mit der ärztlichen Aufklärung. Insgesamt 20\% der Patienten gaben an, dass die anti-infektiösen Supportivmaßnahmen eine Belastung darstellten.

Kommentar: Die Untersuchung unterstreicht mehrere wichtige Punkte zur antiinfektiösen Prophylaxe: Zum einen werden die verschiedenen Maßnahmen von den einzelnen Patienten unterschiedlich gut befolgt, wodurch eine Einschätzung zur Wertigkeit sehr schwierig wird. Weiterhin werden die Prophylaxemaßnahmen nicht einheitlich von allen Kliniken empfohlen, was für viele Patienten sehr verwirrend ist. Letztlich stellen die Maßnah- men für etwa ein Fünftel der Patienten eine zusätzliche und möglicherweise unnötige Belastung dar. Aus all diesen Gründen sind Studien notwendig, die den Sinn von den verschiedenen Prophylaxemaßnahmen untersuchen, um diese dann einheitlich zu empfehlen, wenn durch sie die Infekt-assoziierte Morbidität und möglicherweise auch die Mortalität gesenkt werden kann.

Prof. Dr. Thomas Lehrnbecher

Lehrnbecher T et al. Compliance with antiinfective preventive measures: A multicentre survey among paediatric oncology patients. Eur J Cancer 2008; 44: 1861-5

\title{
Schützt das Landleben auch im Alter?
}

\section{Wer auf dem Bauernhof aufwächst, hat ein niedrigeres Allergierisiko. So weit, so gut. Aber wie lange hält dieser Effekt an? Um dies herauszufinden, wurden in einer Populationsstudie über 18.000 Schweden befragt.}

- - in ine Arbeitsgruppe um Jonas Eriksson befragten für ihre Studie Jugendliche und Erwachsene, die zu gleichen Teilen aus Göteborg oder aus anderen Regionen in West-Schweden stammten. Es wurden vier Altersgruppen gebildet: von 16-30, 31-45, 46-60 und 61-75 Jahre. Diejenigen, die in den ersten fünf Lebensjahren auf einem Bauernhof gelebt hatten, zeigten ein signifikant geringeres Risiko für eine allergische Rhinitis als solche ohne Kindheit auf dem Lande. Dieser Effekt war in der Gruppe der 16bis 30-Jährigen am deutlichsten (19,5 vs. $30,5 \%, \mathrm{p}<0,001)$. Er konnte jedoch auch noch bei Personen zwischen 61 und 75 Jahren nachgewiesen werden (16,5 vs. $19,4 \%, \mathrm{p}=0,045)$.

Die Untersuchung zeigte auch eine signifikante Abhängigkeit der Häufigkeit allergischer Rhinitiden von der Urbanisierung. Menschen in der Großstadt (Göteborg) und anderen mittelgroßen Städten waren häufiger betroffen als Bewohner von Kleinstädten oder ländlichen Regionen. Allerdings zeigte sich hier in der Subgruppe der 61- bis 75-Jährigen kein signifikanter Unterschied mehr.

Kommentar: Mit dieser aufwendigen Befragung bei über 18.000 Personen zeigt sich, dass ein Aufwachsen auf dem Bau-

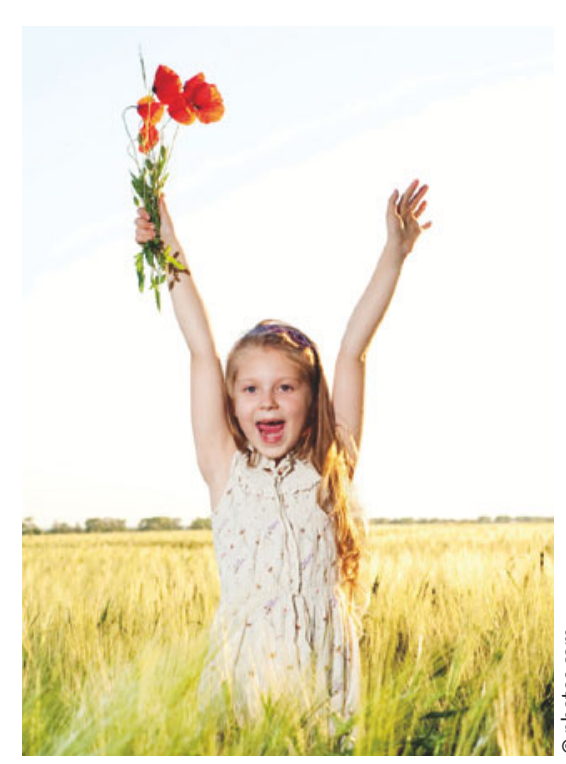

Grund zum Jubeln: Wer auf dem Bauernhof aufwächst, profitiert auch noch im Alter davon.

ernhof einen schützenden Effekt auf die Entwicklung einer allergischen Rhinitis hat, der noch bis ins hohe Alter erkennbar ist.

Dr. Ulrich Mutschler

Eriksson J et al. Growing up on a farm leads to lifelong protection against allergic rhinitis. Allergy 2010 65: 1397-403 